\title{
Feasibility of further expansion of the indications for endoscopic submucosal dissection in undifferentiated-type early gastric cancer
}

\author{
Yusuke Horiuchi ${ }^{1}(0)$ Satoshi Ida ${ }^{2} \cdot$ Noriko Yamamoto $^{3} \cdot$ Souya Nunobe $^{2} \cdot$ Naoki Ishizuka $^{4} \cdot$ Shoichi Yoshimizu $^{1}$. \\ Akiyoshi Ishiyama ${ }^{1} \cdot$ Toshiyuki Yoshio $^{1} \cdot$ Toshiaki Hirasawa $^{1} \cdot$ Tomohiro Tsuchida $^{1} \cdot$ Koshi Kumagai $^{2}$. \\ Manabu Ohashi ${ }^{2} \cdot$ Takeshi Sano $^{2} \cdot$ Junko Fujisaki $^{1}$
}

Received: 27 July 2019 / Accepted: 27 August 2019 / Published online: 5 September 2019

(c) The International Gastric Cancer Association and The Japanese Gastric Cancer Association 2019

\begin{abstract}
Background Based on Japanese guidelines for endoscopic submucosal dissection (ESD) in undifferentiated-type early gastric cancer (UD-EGC), UD-predominant mixed-type (M-UD) EGC is considered high risk for lymph node metastasis (LNM). However, differences in LNM risk between pure UD (P-UD) and M-UD remain unclear. This study assessed risk factors for LNM considering differences between P-UD and M-UD and identified pathological features related to the lowest LNM risk. Methods This single-center, retrospective study included 1425 patients with UD-EGC treated with surgical resection between April 2005 and May 2017. We divided patients into those with and without LNM and compared background characteristics and post-operative pathological results between groups. Patients were further stratified based on depth, tumor diameter, ulcerative findings, lymphatic invasion, vascular invasion, and histological type to clarify post-operative pathological features associated with the lowest LNM risk.

Results When comparing background characteristics and post-operative pathological results, multivariate analysis showed that, in patients with LNM, tumor diameters were significantly larger, and there were higher rates of submucosal invasion, lymphatic invasion, and M-UD histological type. In patients with absence of ulcerative findings, absence of lymphatic invasion, and absence of vascular invasion, no LNM occurred among those with intramucosal P-UD tumor diameters of 1-40 mm (1-20 mm: 95\% confidence interval [CI], 0-5.5\%; 21-40 mm: 95\% CI, 0-6.1\%).

Conclusions Intramucosal P-UD EGC patients with absence of ulcerative findings, absence of lymphatic invasion, absence of vascular invasion, and tumor diameters of $\leq 40 \mathrm{~mm}$ did not show LNM. We suggest expanding indications for ESD to include these patients.
\end{abstract}

Keywords Gastric cancer $\cdot$ Endoscopic submucosal resection $\cdot$ Gastric carcinoma $\cdot$ Lymph node metastasis

\section{Introduction}

Yusuke Horiuchi

yusuke.horiuchi@jfcr.or.jp

1 Department of Gastroenterology, Cancer Institute Hospital, 3-10-6 Ariake, Koto-ku, Tokyo 135-8550, Japan

2 Department of Gastric Surgery, Cancer Institute Hospital, 3-10-6 Ariake, Koto-ku, Tokyo 135-8550, Japan

3 Department of Pathology, Cancer Institute Hospital, 3-10-6 Ariake, Koto-ku, Tokyo 135-8550, Japan

4 Department of Clinical Trial Planning and Management, Cancer Institute Hospital, 3-10-6 Ariake, Koto-ku, Tokyo 135-8550, Japan
Since the development of endoscopic submucosal resection (ESD), endoscopic resection has been possible for early gastric cancers (EGC) with a large tumor diameter. Therefore, the treatment of EGC has become less invasive [1-6]. However, because all undifferentiated-type EGCs (UDEGC) carry a high risk of lymph node metastasis (LNM), traditional surgical treatment remains the current standard treatment according to Japanese gastric cancer treatment guidelines [7].

However, Hirasawa et al. stratified intramucosal UDEGC patients who underwent surgical resection based on tumor diameter, presence or absence of lymphatic invasion and vascular invasion, and presence or absence of ulcerative 
findings [8]. Among patients with absence of ulcerative findings, absence of lymphatic invasion, and absence of vascular invasion, none showed LNM in tumors with a diameter of $\leq 20 \mathrm{~mm}$ [8]. Therefore, tumors with a diameter of $\leq 20 \mathrm{~mm}$ and no LNM are listed in the Japanese gastric cancer treatment guidelines 2014 (ver. 4) as indicated for ESD as an investigational treatment (expanded indication) [7]. Two reports on the long-term prognosis after ESD in patients with these lesions have reported a 5-year survival rate of approximately $100 \%[9,10]$. However, there have also been reports of no significant differences in 5-year survival rates between patients who undergo surgery and those who undergo ESD [11].

A multicenter collaborative prospective study on the long-term prognosis of tumors of $\leq 20 \mathrm{~mm}$ in diameter, with the absence of ulcerative findings and absence of lymphatic invasion and vascular invasion and intramucosal UD-EGC (JCOG1009/1010), is in progress [12]. If the long-term prognosis in this study is good, it may support the use of ESD as the standard treatment for these lesions. Furthermore, further expansions of the indications for ESD in UD-EGC may be feasible.

Notably, Takizawa et al. and Hanaoka et al. examined surgical specimens of UD-EGCs and found that the risk of LNM was higher in undifferentiated-type predominant mixed-type (M-UD) cancers than in pure undifferentiatedtype cancers (P-UD) $[13,14]$. However, according to the Japanese guidelines [7], P-UD and M-UD are collectively treated as UD-EGC. Consequently, differences in LNM risk between P-UD and M-UD have not been given much consideration. Therefore, the aim of this study was to compare the background characteristics and post-operative pathological results in patients with and without LNM. We also aimed to clarify the post-operative pathological features of patients with the lowest risk of LNM by stratification based on possible influential variables, including P-UD and M-UD. These data could be used to assess the feasibility of further expansion of the indications for ESD in UD-EGC.

\section{Methods}

This was a single-center, retrospective, observational study. We extracted patient data from electronic medical records of patients who met the following inclusion criteria: (1) patients with UD-EGC (intramucosal or submucosal cancer) who underwent surgery as the initial treatment at the Cancer Institute Hospital between April 2005 and May 2017, (2) patients with positive post-operative UD-EGC results on pathological tests, (3) patients with a single lesion, and (4) patients in whom pathological results could be collected retrospectively.
The exclusion criteria were as follows: (1) patients with remnant cancer or gastric tube cancer, (2) patients with multiple lesions, (3) patients who had undergone chemotherapy and/or radiation, and (4) patients in whom ESD had already been performed.

We first clarified the backgrounds of the patients (age and sex) and determined their post-operative pathological results (location of tumor, depth, tumor diameter, presence or absence of ulcerative findings, presence or absence of lymphatic invasion and vascular invasion, LNM, and histological type). We then divided patients into two groups based on the presence or absence of LNM. Background characteristics and post-operative pathological results were compared between groups to identify significant risk factors for LNM. The cutoff value for age was set to the median of the overall study population. Moreover, to the best of our knowledge, there have been no reports regarding detailed examination of lesions $>20 \mathrm{~mm}$; therefore, the cutoff value for tumor diameter was set to the median for cases with presence of LNM because many lesions were presumed to be $>20 \mathrm{~mm}$ in cases with LNM. Following the clarification of the post-operative pathological features of patients with the lowest risk of LNM, we performed stratification based on possible influential variables according to the results of univariate analysis.

All patients with UD-EGC who were treated with surgery underwent pretreatment conventional endoscopy, dye-spraying endoscopy, and magnifying endoscopy with narrowband imaging to determine the extent and depth of the tumor. Before the treatment, the advantages and disadvantages of the surgery were fully explained to each patient, and each patient provided informed consent to undergo surgery. This study was approved by the institutional review board (IRB) of the Cancer Institute Hospital (IRB number: 2017-1068), and it conformed to the provisions of the Declaration of Helsinki (as revised in Fortaleza, Brazil, October 2013).

\section{Pathological validation}

For each surgically resected specimen, the sections were prepared at 5-mm intervals for pathological evaluation. Pathologists who specialized in gastrointestinal pathology performed all pathological examinations. The measurements and assessments undertaken were maximum tumor diameter, invasion depth, histological type, presence or absence of ulcerative findings (ulcers and scars that extend deep into the submucosa), lymphatic invasion, vascular invasion, oral margin, anal margin, and presence or absence of LNM. Immunostaining on histological assessment was performed to explore lymphatic invasion (by D2-40 staining) and vascular invasion (by Victoria blue hematoxylin-eosin staining) when the depth was indicative of submucosal cancer or when lymphovascular invasion was suspected based on 
hematoxylin-eosin staining. R0 (no cancer in any resection margins) surgical patients were considered curative resection patients based on the Japanese guidelines [7]. All patients were classified into P-UD or M-UD cancer groups based on the post-operative pathological results. A case of P-UD was defined as a case that only had the UD component. A case of M-UD was defined as a case in which the UD component exceeded $50 \%$ of the lesion with a differentiated-type component.

Based on the Japanese guidelines, the UD components comprised poorly differentiated adenocarcinoma, signet ring cell carcinoma, and mucinous adenocarcinoma, while the differentiated-type components comprised moderately or well-differentiated adenocarcinoma and papillary adenocarcinoma.

\section{Statistical analysis}

The chi-squared test was used to compare the two groups, based on the presence or absence of LNM. The $F$ test was performed to determine whether distributions exhibited equal variance between the two groups for age and tumor diameter. If the $F$ test showed significance, the median, interquartile range, and overall range by age and tumor diameter were calculated and analyzed by Mann-Whitney $U$ test. If the $F$ test showed no significance, the mean, standard deviation by age, and tumor diameter were calculated and analyzed by $t$ test. When comparing patients with and without LNM, a multivariate analysis (logistic regression analysis) was performed on variables that showed significant differences in the univariate analysis described above. The odds ratio and 95\% confidence interval (CI) were calculated. In addition, we calculated the rate and 95\% CI of LNM in patients based on possible influential variables identified in univariate analysis. Statistical significance was set at $p<0.05$ for both univariate and multivariate analyses. JMP software, version 13.2 (SAS ${ }^{\circledR}$ Institute Inc., Cary, NC, USA), was used for statistical analyses.

\section{Results}

A total of 1844 UD-EGC patients who received initial treatment (ESD: 361 patients, surgery: 1483 patients) at the Cancer Institute Hospital between April 2005 and May 2017 were initially enrolled in this study. After exclusion criteria were applied, 1425 patients and 1425 UD-EGC lesions were included in the analysis (Fig. 1). Background characteristics and post-operative pathological results are shown in Table 1. The median age of the patients was 60 years. The median tumor diameter was $30 \mathrm{~mm}$ (interquartile range: 20-43.5 mm). LNM was present in $170(11.9 \%)$ patients and absent in $1255(88.1 \%)$ patients.

The prevalence of submucosal invasion, the presence of lymphatic invasion, the presence of vascular invasion, the presence of ulcerative findings, and the prevalence of M-UD type were significantly higher in the LNM group (Table 2). The median tumor diameter of cases with LNM was $40 \mathrm{~mm}$. Tumor diameters were significantly larger $(>40 \mathrm{~mm})$ in the LNM group.

Tumor diameter of $>40 \mathrm{~mm}$ was significantly associated with LNM (Table 3). Submucosal invasion, presence of lymphatic invasion, and prevalence of M-UD were also significantly higher in patients with LNM (Table 3).

Based on the above results, intramucosal UD EGC patients were stratified based on tumor diameter, histological
Fig. 1 Flowchart of patient inclusion and exclusion. ESD endoscopic submucosal resection

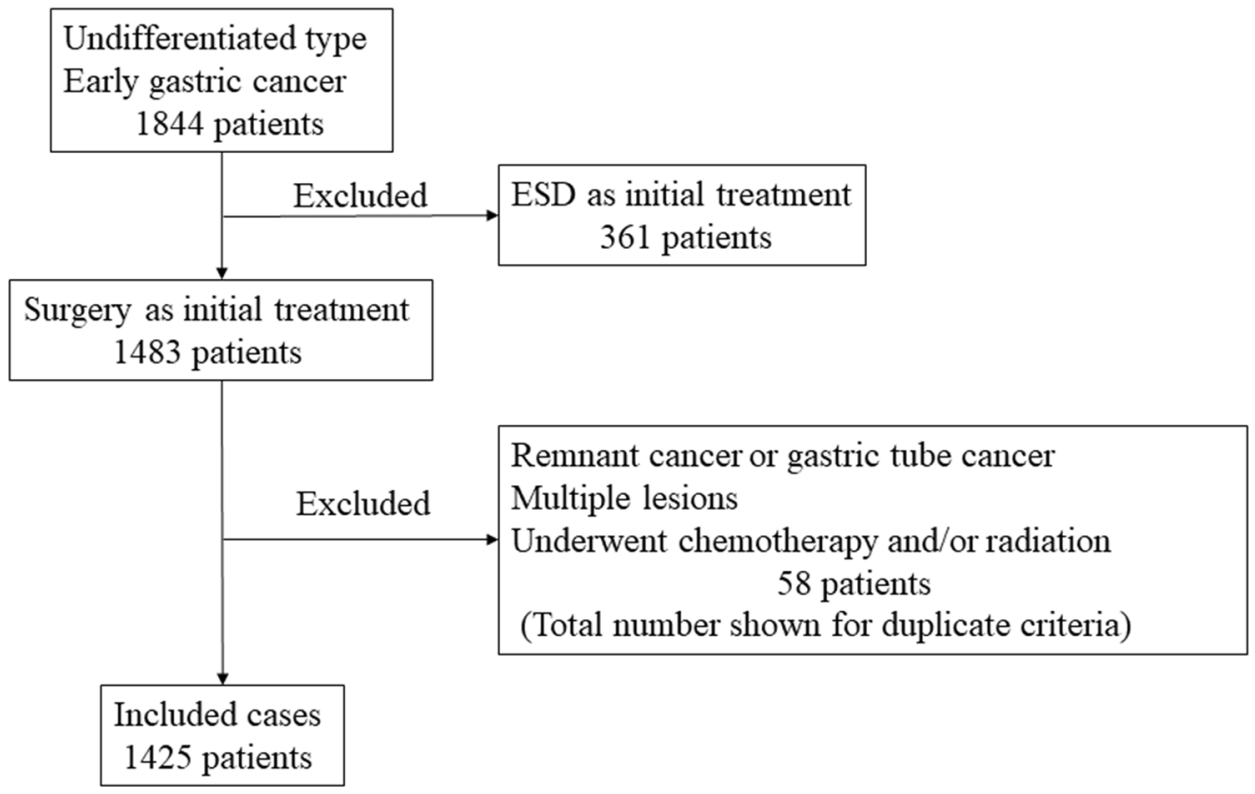


Table 1 Background characteristics and post-operative pathological results of patients

1425 cases, 1425 lesions

\begin{tabular}{ll}
\hline Age (years) & $60(50-68)[19-91]^{\mathrm{a}}$ \\
Median & \\
Sex & $711(49.9)$ \\
Male & $714(50.1)$ \\
Female & \\
Location & $208(14.6)$ \\
Upper third & $939(65.9)$ \\
Middle third & $278(19.5)$ \\
Lower third & \\
Depth of invasion & $707(49.6)$ \\
Intramucosal cancer & $718(50.4)$ \\
Submucosal cancer & \\
Tumor diameter (mm) & $30(20-43.5)[2-140]^{\mathrm{a}}$ \\
Median & \\
Ulcerative findings & $353(24.7)$ \\
Presence & $1072(75.2)$ \\
Absence & \\
Lymphatic invasion & $294(20.6)$ \\
Presence & $1131(79.4)$ \\
Absence & \\
Vascular invasion & $159(11.2)$ \\
Presence & $1266(88.8)$ \\
Absence & \\
Histological type & \\
Pure undifferentiated-type & \\
Undifferentiated-type predominant & $525(36.8)$ \\
LNM & \\
Presence & \\
Absence & \\
\hline
\end{tabular}

LNM lymph node metastasis

${ }^{a}$ Data are presented as number (\%), except age and tumor diameter, which are expressed as median (interquartile range) [range]

${ }^{\mathrm{b}}$ Pure undifferentiated-type: poorly differentiated adenocarcinoma and/or signet ring cell carcinoma, and/or mucinous adenocarcinoma

${ }^{c}$ Undifferentiated-type predominant mixed-type: poorly differentiated adenocarcinoma and/or signet ring cell carcinoma, and/or mucinous adenocarcinoma with differentiated-type components comprising moderately differentiated or well-differentiated adenocarcinoma and papillary adenocarcinoma

type, presence or absence of lymphatic invasion, the presence or absence of vascular invasion, and the presence or absence of ulcerative findings in which significant differences were found in univariate analysis (Table 4). In patients with absence of ulcerative findings, absence of lymphatic invasion, and absence of vascular invasion, LNM did not occur for P-UD tumors with a diameter of $1-40 \mathrm{~mm}$ or for M-UD tumors with a diameter of $1-30 \mathrm{~mm}$. However, there were fewer M-UD tumors than P-UD tumors. In the other groups, although the tumor diameter was 1-20 mm, LNM was positive; alternatively, because the number of cases was one or two, it was difficult to interpret the results.

We calculated the rate of LNM and the $95 \% \mathrm{CI}$ in patients with absence of ulcerative findings, absence of lymphatic invasion, and absence of vascular invasion (Table 5). The 95\% CI was 0-6.1\% for P-UD patients without LNM, a tumor diameter of 21-40 mm, absence of ulcerative findings, absence of lymphatic invasion, and absence of vascular invasion.

\section{Discussion}

In this study, we stratified cases of intramucosal UD-EGC based on tumor diameter, histological type, presence or absence of lymphatic invasion, presence or absence of vascular invasion, and presence or absence of ulcerative findings to clarify the post-operative pathological features of patients with the lowest risk of LNM. To the best of our knowledge, our study is the first detailed investigation of the proportion of LNM that included stratification of tumors by diameter, with consideration of the histological type (P-UD and M-UD).

In this study, the independent risk factors for LNM in UDEGC were submucosal invasion, lymphatic invasion, M-UD, and a tumor diameter of $>40 \mathrm{~mm}$. With the exception of one lesion, vascular invasion was also present in lesions with submucosal invasion, and/or lymphatic invasion. Therefore, although there was a significant difference regarding vascular invasion in univariate analysis, there was no significant difference in multivariate analysis. Submucosal invasion and lymphatic invasion have been previously reported as factors that are significantly correlated with LNM [8, 15-18], and the results of this study were similar to those of other reports in the literature. Takizawa et al. reported that M-UD was a significant risk factor for LNM in intramucosal UD-EGC [13] and Hanaoka et al. reported similar results for submucosal invasive UD-EGC [14]; our results are compatible with these previously reported results. However, the factors underlying the high rates of LNM in intramucosal M-UD patients with absence of ulcerative findings, absence of lymphatic invasion, and absence of vascular invasion were not clarified in the previous reports. Since pathological sections were prepared at 5-mm intervals for pathological evaluation, evidence of lymphatic invasion and vascular invasion may have been concealed within these sections. Therefore, if pathological sections are prepared at $<5$-mm intervals, the numbers of intramucosal cases with presence of lymphatic and/or vascular invasion may increase. In the future, differences in pathology analysis should be considered.

A tumor diameter of $>20 \mathrm{~mm}$ has been reported as a significant risk factor for LNM [8, 15, 19], and the results 
Table 2 Results of chi-squared test and $t$ test comparisons of the background of patients and post-operative pathological results based on the presence or absence of LNM

\begin{tabular}{|c|c|c|c|}
\hline & Presence of $\mathrm{LNM}^{\mathrm{a}}(n=170)$ & Absence of LNM $(n=1255)$ & $p$ value $^{\mathrm{b}}$ \\
\hline \multicolumn{4}{|l|}{ Age (years) } \\
\hline Mean \pm SD & $58.7 \pm 12.4^{\mathrm{c}}$ & $58.5 \pm 12.9$ & 0.8327 \\
\hline$>60$ years & $78(45.9)$ & $586(47.0)$ & 0.8423 \\
\hline$\leq 60$ years & $92(54.1)$ & $669(53.3)$ & \\
\hline \multicolumn{4}{|l|}{ Sex } \\
\hline Male & $82(48.2)$ & $629(49.9)$ & 0.6447 \\
\hline Female & $88(51.8)$ & $626(50.1)$ & \\
\hline \multicolumn{4}{|l|}{ Location } \\
\hline Upper third & $22(12.9)$ & $186(14.8)$ & 0.2567 \\
\hline Middle third & $107(62.9)$ & $832(66.3)$ & \\
\hline Lower third & $41(24.1)$ & $237(18.9)$ & \\
\hline \multicolumn{4}{|l|}{ Depth } \\
\hline Intramucosal cancer & $27(15.9)$ & $680(54.2)$ & $<0.0001^{*}$ \\
\hline Submucosal cancer & $143(84.1)$ & $575(45.8)$ & \\
\hline \multicolumn{4}{|l|}{ Tumor diameter (mm) } \\
\hline Median & $40(26-52)[5-140]^{\mathrm{c}}$ & $28(20-41)[2-135]$ & $<0.0001^{*}$ \\
\hline$\leq 40 \mathrm{~mm}$ & $82(48.2)$ & $318(25.3)$ & $<0.0001^{*}$ \\
\hline$>40 \mathrm{~mm}$ & $88(51.8)$ & $937(74.7)$ & \\
\hline \multicolumn{4}{|l|}{ Ulcerative findings } \\
\hline Absence & $32(18.8)$ & $321(25.6)$ & $0.0494 *$ \\
\hline Presence & $138(81.2)$ & $934(74.4)$ & \\
\hline \multicolumn{4}{|l|}{ Lymphatic invasion } \\
\hline Absence & $69(40.6)$ & $1062(84.6)$ & $<0.0001^{*}$ \\
\hline Presence & $101(59.4)$ & $193(15.4)$ & \\
\hline \multicolumn{4}{|l|}{ Vascular invasion } \\
\hline Absence & $127(74.7)$ & $1139(90.8)$ & $<0.0001^{*}$ \\
\hline Presence & $43(25.3)$ & $116(9.2)$ & \\
\hline \multicolumn{4}{|l|}{ Histological type } \\
\hline Pure undifferentiated-type ${ }^{d}$ & $66(38.8)$ & $834(66.5)$ & $<0.0001^{*}$ \\
\hline $\begin{array}{l}\text { Undifferentiated predomi- } \\
\text { nant mixed-type }\end{array}$ & $104(61.2)$ & $421(33.6)$ & \\
\hline
\end{tabular}

${ }^{\mathrm{a}} L N M$ lymph node metastasis

${ }^{\mathrm{b}}$ The $t$ test was used to compare mean \pm standard deviation (SD) values for age after confirming non-significance by $F$ test. The Mann-Whitney $U$ test was used to compare median values for tumor diameter after confirming significance by $F$ test. The chi-squared test was used to compare the presence and absence of LNM groups in terms of age ( $>60$ years or $\leq 60$ years), sex, location, depth, tumor diameter $(<40 \mathrm{~mm}$ or $\geq 40 \mathrm{~mm}$ ), ulcerative findings, lymphatic invasion, vascular invasion, and histological type

${ }^{\mathrm{c}}$ Data are presented as number (\%), except age, which is expressed as mean $\pm \mathrm{SD}$, and tumor diameter, which is expressed as median (interquartile range) [range]

${ }^{\mathrm{d}}$ Pure undifferentiated-type: poorly differentiated adenocarcinoma and/or signet ring cell carcinoma, and/or mucinous adenocarcinoma,

${ }^{\mathrm{e}}$ Undifferentiated-type predominant mixed-type: poorly differentiated adenocarcinoma and/or signet ring cell carcinoma, and/or mucinous adenocarcinoma with differentiated-type components comprising moderately differentiated or well-differentiated adenocarcinoma and papillary adenocarcinoma

*Statistically significant of this study are consistent with those of previous reports. We stratified intramucosal UD-EGC patients based on tumor diameter, presence or absence of lymphatic invasion and vascular invasion, histological type (P-UD or M-UD), and presence or absence of ulcerative findings. Hirasawa et al. stratified intramucosal UD carcinoma patients who underwent surgical resection [8]. However, the results of their report did not include data regarding histological type (P-UD or M-UD). Because we included histological type (P-UD or M-UD) stratification in our analysis, our data are more detailed than the data described by Hirasawa et al. in terms of histological type. 
Table 3 Logistic regression analysis for comparison of patient background and post-operative pathological results based on the presence or absence of LNM

\begin{tabular}{|c|c|c|c|c|c|c|c|c|}
\hline & Odds ratio & $95 \% \mathrm{CI}^{\mathrm{a}}$ & $p$ value & Post-operative pathological & P-UD & LNM & M-UD & LNM \\
\hline \multicolumn{4}{|l|}{ Depth } & & & & & \\
\hline Intramucosal cancer & 1 & \multirow{3}{*}{$1.5-4.2$} & \multirow{3}{*}{$0.0003^{*}$} & 1-20 mm UL -, LVI- & 66 & 0 & 14 & 0 \\
\hline Submucosal cancer & 2.5 & & & 21-30 mm UL -, LVI- & 36 & 0 & 9 & 0 \\
\hline \multicolumn{2}{|l|}{ Tumor diameter } & & & 31-40 mm UL -, LVI - & 23 & 0 & 10 & $1(10.0)^{\mathrm{a}}$ \\
\hline$\leq 40 \mathrm{~mm}$ & 1 & \multirow[b]{2}{*}{$1.8-3.6$} & \multirow[b]{2}{*}{$<0.0001^{*}$} & 41-50 mm UL -, LVI- & 13 & $1(7.7)$ & 8 & $1(11.1)$ \\
\hline$>40 \mathrm{~mm}$ & 2.5 & & & $>50 \mathrm{~mm} \mathrm{UL}-, \mathrm{LVI}-$ & 10 & $1(10.0)$ & 9 & 0 \\
\hline \multicolumn{4}{|l|}{ Ulcerative findings } & 1-20 mm UL +, LVI - & 110 & $3(2.7)$ & 30 & $1(3.3)$ \\
\hline Absence & 1 & \multirow{3}{*}{$0.9-2.1$} & \multirow{3}{*}{0.1587} & 21-30 mm UL,+ LVI - & 97 & $2(2.1)$ & 38 & 0 \\
\hline \multirow{2}{*}{\multicolumn{2}{|c|}{ Lymphatic invasion }} & & & 31-40 mm UL +, LVI- & 61 & $2(3.3)$ & 24 & $1(4.2)$ \\
\hline & & & & 41-50 mm UL +, LVI- & 48 & $2(4.2)$ & 17 & $2(11.8)$ \\
\hline $\begin{array}{l}\text { Absence } \\
\text { Presence }\end{array}$ & $\begin{array}{l}1 \\
4.8\end{array}$ & \multirow{2}{*}{$3.1-7.3$} & \multirow{2}{*}{$<0.0001^{*}$} & $>50 \mathrm{~mm} \mathrm{UL}+, \mathrm{LVI}-$ & 41 & 0 & 34 & $9(26.5)$ \\
\hline \multicolumn{2}{|l|}{ Vascular invasion } & & & $1-20 \mathrm{~mm} \mathrm{UL}-, \mathrm{LVI}+$ & 0 & 0 & 0 & 0 \\
\hline Absence & 1 & \multirow{3}{*}{$0.7-1.7$} & \multirow{3}{*}{0.8632} & 21-30 mm UL -, LVI+ & 0 & 0 & 0 & 0 \\
\hline Presence & 1.0 & & & 31-40 mm UL -, LVI+ & 1 & $1(100)$ & 0 & 0 \\
\hline \multicolumn{2}{|l|}{ Histological type } & & & 41-50 mm UL -, LVI+ & 0 & 0 & 0 & 0 \\
\hline Pure undifferentiated-type ${ }^{b}$ & 1 & \multirow{3}{*}{$1.4-2.9$} & \multirow{3}{*}{$0.0002^{*}$} & $>50 \mathrm{~mm} \mathrm{UL}-, \mathrm{LVI}+$ & 1 & 0 & 0 & 0 \\
\hline \multirow{2}{*}{$\begin{array}{l}\text { Undifferentiated-type pre- } \\
\text { dominant mixed-type }{ }^{\mathrm{c}}\end{array}$} & 2.0 & & & 1-20 mm UL +, LVI+ & 2 & 0 & 0 & 0 \\
\hline & & & & 21-30 mm UL +, LVI+ & 2 & 0 & 0 & 0 \\
\hline \multicolumn{4}{|l|}{${ }^{\mathrm{a}} \mathrm{CI}$ confidence interval } & 31-40 mm UL,+ LVI + & 2 & 0 & 0 & 0 \\
\hline \multirow{2}{*}{\multicolumn{4}{|c|}{$\begin{array}{l}{ }^{b} \text { Pure undifferentiated-type: poorly differentiated adenocarcinoma } \\
\text { and/or signet ring cell carcinoma, and/or mucinous adenocarcinoma }\end{array}$}} & $41-50 \mathrm{~mm} \mathrm{UL}+, \mathrm{LVI}+$ & 1 & $1(100)$ & 0 & 0 \\
\hline & & & & $>50 \mathrm{~mm} \mathrm{UL}+, \mathrm{LVI}+$ & 0 & 0 & 0 & 0 \\
\hline
\end{tabular}

${ }^{c}$ Undifferentiated-type predominant mixed-type: poorly differentiated adenocarcinoma and/or signet ring cell carcinoma, and/or mucinous adenocarcinoma with differentiated-type components comprising moderately differentiated or well-differentiated adenocarcinoma and papillary adenocarcinoma

*Statistically significant

Sekiguchi et al. [19] validated a risk scoring model for predicting LNM based on tumor size, invasion depth, histological type, presence or absence of ulcerative findings, and presence or absence of lymphatic invasion and vascular invasion in all cases of EGC. They reported higher rates of lymphatic invasion, vascular invasion, and submucosal invasion; large tumor diameters; and higher prevalence of ulcerative findings and M-UD in patients with LNM [19]. However, because the cutoff value of the tumor diameter was set to $20 \mathrm{~mm}$ in that study, detailed examination of lesions larger than $20 \mathrm{~mm}$ was not performed [19]. Therefore, because we have adopted a 40-mm cutoff value with regard to the histological type, our data are more detailed than the data described by Sekiguchi et al. in terms of tumor diameter assessment.

The results of our study suggest for the first time that LNM does not occur in intramucosal P-UD carcinoma patients with absence of ulcerative findings, absence of lymphatic invasion, absence of vascular invasion, and a tumor diameter of $\leq 40 \mathrm{~mm}$. The $95 \% \mathrm{CI}$ for P-UD patients without
Table 4 Stratification based on tumor diameter, histological type, presence or absence of LVI, and presence or absence of ulcerative findings in intramucosal undifferentiated-type carcinoma

$U L$ ulcerative findings, $L V I$ lymphatic invasion and/or vascular invasion, $P-U D$ pure undifferentiated-type, $M-U D$ undifferentiated-type predominant mixed-type, $L N M$ lymph node metastasis

${ }^{\mathrm{a}}$ Data are presented as number (\%)

LNM, a tumor diameter of $21-40 \mathrm{~mm}$, absence of ulcerative findings, absence of lymphatic invasion, and absence of vascular invasion was $0-6.1 \%$. Based on the current Japanese gastric cancer treatment guidelines [7], less than $1 \%$ of $95 \% \mathrm{CI}$ is regarded as within expanded indications for ESD. Therefore, the upper limit of the $95 \%$ CI of the LNM rate was high. However, based on the results of this study, we suggest expansion of the indications for ESD to include patients with an endoscopic tumor diameter of $\leq 40 \mathrm{~mm}$, absence of endoscopic ulcerative findings, absence of endoscopic submucosal invasion, and P-UD on biopsy (Fig. 2). In curative evaluation, in P-UD carcinomas in which R0 resection was performed by ESD, we suggest expansion of the indications for curative resection to include patients with a pathological tumor diameter of $\leq 40 \mathrm{~mm}$, absence of pathological ulcerative findings, absence of pathological submucosal invasion, absence of lymphatic invasion, and absence of vascular invasion. Moreover, the frequency of serious surgery-related deaths and early post-operative deaths is reportedly higher in elderly patients [20-22]. Therefore, 
Table 5 LNM rates and 95\% CIs in the group of patients without ulcerative findings and LVI

\begin{tabular}{lllllll}
\hline Post-operative pathological result & P-UD & LNM & LNM 95\% CI (\%) & M-UD & LNM & LNM 95\% CI (\%) \\
\hline 1-20 mm, UL-, LVI- & 66 & 0 & $0-5.5$ & 14 & 0 & $0-21.5$ \\
21-40 mm, UL-, LVI- & 59 & 0 & $0-6.1$ & 19 & $1(5.3)^{\mathrm{a}}$ & $0.9-24.6$ \\
$>41 \mathrm{~mm}$, UL-, LVI- & 23 & $2(8.7)$ & $2.4-26.8$ & 17 & $1(5.9)$ & $1.0-27.0$ \\
\hline
\end{tabular}

$U L$ ulcerative findings, $L V I$ lymphatic invasion and/or vascular invasion, $P$ - $U D$ pure undifferentiated-type, $M-U D$ undifferentiated-type predominant mixed-type, $L N M$ lymph node metastasis, $C I$ confidence interval

${ }^{\mathrm{a}}$ Data are presented as number $(\%)$
Fig. 2 Extended criteria for endoscopic submucosal resection in undifferentiated-type early gastric cancer. Cases of endoscopic tumor with a diameter of $<40 \mathrm{~mm}$, absence of endoscopic ulcerative findings, absence of pathological invasion depth, and pure undifferentiated-type carcinoma classification on biopsy are suggested as additions for indications for ESD. ESD endoscopic submucosal resection, $U L$ ulcerative findings, $P$ - $U D$ pure undifferentiated-type, $M-U D$ undifferentiated-type predominant mixed-type

\begin{tabular}{|c|c|c|c|c|c|}
\hline \multirow[b]{2}{*}{ Histological type } & & \multicolumn{4}{|c|}{ Intramucosal cancer } \\
\hline & & $\leq 20 \mathrm{~mm}$ & $\begin{array}{c}\text { UL- } \\
>\mathbf{2 0} \text { to } \leq 40 \\
\mathrm{~mm}\end{array}$ & $>40 \mathrm{~mm}$ & $\begin{array}{c}\text { UL+ } \\
\text { Any size }\end{array}$ \\
\hline Undifferentiated type & $\begin{array}{l}\text { P-UD } \\
\text { M-UD }\end{array}$ & & & & \\
\hline
\end{tabular}

Current extended criteria for ESD

\section{Candidates for new extended criteria for ESD}

\section{Surgery}

pathological evaluation, while ESD specimens were prepared at 2-mm intervals. Therefore, it is also necessary to prospectively examine long-term prognosis to establish ESD as standard treatment for post-ESD patients who meet the above criteria. However, given that this study included large number of UD-EGC surgery cases over a period of 12 years at a cancer specialty hospital, our results are sufficiently meaningful in that they can serve as the basis for the feasibility of expanded indications for ESD when evaluating subjects for multicenter prospective studies.

In conclusion, our findings suggest that intramucosal P-UD EGC patients with absence of ulcerative findings, absence of lymphatic invasion, absence of vascular invasion, and tumor diameters of $\leq 40 \mathrm{~mm}$ have the lowest risk of LNM. We suggest including these patients in the expanded indications for curative resection of ESD in UD-EGC. In the future, our results can be the basis for subject selection in multicenter, prospective studies. 
Author contributions Conceptualization: YH, SI, SN, NY, JF; methodology: YH, SI, NI; formal analysis and investigation: YH, NI; writing — original draft preparation: $\mathrm{YH}$; writing — review and editing: $\mathrm{YH}$, TY, KK, NI, SN; funding acquisition: YH; resources: YH, SI, SN, SY, AI, TY, TH, TT, KK, MO, TS, JF; supervision: YH, JF.

\section{Compliance with ethical standards}

Conflict of interest There is no conflict of interest associated with this study.

Human rights statement All procedures followed were in accordance with the ethical standards of the responsible committee on human experimentation (institutional and national) and with the Helsinki Declaration of 1964 and later versions.

Informed consent Informed consent to be included in the study, or the equivalent, was obtained from all patients.

\section{References}

1. Ono H, Kondo H, Gotoda T, Shirao K, Yamaguchi H, Saito D, et al. Endoscopic mucosal resection for treatment of early gastric cancer. Gut. 2001;48:225-9.

2. Oda I, Gotoda T, Hamanaka H, Eguchi T, Saito Y, Matsuda T, et al. Endoscopic submucosal dissection for early gastric cancer: technical feasibility, operation time and complications from a large consecutive series. Dig Endosc. 2005;17:54-8.

3. Ono H, Hasuike N, Inui T, Takizawa K, Ikehara H, Yamaguchi $\mathrm{Y}$, et al. Usefulness of a novel electrosurgical knife, the insulationtipped diathermic knife-2, for endoscopic submucosal dissection of early gastric cancer. Gastric Cancer. 2008;11:47-52.

4. Gotoda T, Kondo H, Ono H, Saito Y, Yamaguchi H, Saito D, et al. A new endoscopic mucosal resection procedure using an insulation-tipped electrosurgical knife for rectal flat lesions: report of two cases. Gastrointest Endosc. 1999;50:560-3.

5. Ohkuwa M, Hosokawa K, Boku N, Ohtu A, Tajiri H, Yoshida S. New endoscopic treatment for intramucosal gastric tumors using an insulated-tip diathermic knife. Endoscopy. 2001;33:221-6.

6. Yamamoto H, Kawata H, Sunada K, Satoh K, Kaneko Y, Ido K, et al. Success rate of curative endoscopic mucosal resection with circumferential mucosal incision assisted by submucosal injection of sodium hyaluronate. Gastrointest Endosc. 2002;56:507-12.

7. Japanese Gastric Cancer Association. Japanese gastric cancer treatment guidelines 2014 (ver. 4). Gastric Cancer. 2017;20:1-19.

8. Hirasawa T, Gotoda T, Miyata S, Kato Y, Shimoda T, Taniguchi $\mathrm{H}$, et al. Incidence of lymph node metastasis and the feasibility of endoscopic resection for undifferentiated-type early gastric cancer. Gastric Cancer. 2009;12:148-52.

9. Okada K, Fujisaki J, Yoshida T, Ishikawa H, Suganuma T, Kasuga A, et al. Long-term outcomes of endoscopic submucosal dissection for undifferentiated-type early gastric cancer. Endoscopy. 2012;44:122-7.

10. Abe S, Oda I, Suzuki H, Nonaka S, Yoshinaga S, Odagaki T, et al. Short- and long-term outcomes of endoscopic submucosal dissection for undifferentiated early gastric cancer. Endoscopy. 2013;45:703-7.

11. Lee S, Choi KD, Han M, Na HK, Ahn JY, Jung KW, et al. Longterm outcomes of endoscopic submucosal dissection versus surgery in early gastric cancer meeting expanded indication including undifferentiated-type tumors: a criteria-based analysis. Gastric Cancer. 2018;21:490-9.

12. Takizawa K, Takashima A, Kimura A, Mizusawa J, Hasuike N, Ono $\mathrm{H}$, et al. A phase II clinical trial of endoscopic submucosal dissection for early gastric cancer of undifferentiated type: Japan Clinical Oncology Group study JCOG1009/1010. Jpn J Clin Oncol. 2013;43:87-91.

13. Takizawa K, Ono H, Kakushima N, Tanaka M, Hasuike N, Matsubayashi $\mathrm{H}$, et al. Risk of lymph node metastases from intramucosal gastric cancer in relation to histological types: how to manage the mixed histological type for endoscopic submucosal dissection. Gastric Cancer. 2013;16:531-6.

14. Hanaoka N, Tanabe S, Mikami T, Okayasu I, Saigenji K. Mixedhistologic-type submucosal invasive gastric cancer as a risk factor for lymph node metastasis: feasibility of endoscopic submucosal dissection. Endoscopy. 2009;41:427-32.

15. Gotoda T, Sasako M, Ono H, Katai H, Sano T, Shimoda T. Evaluation of the necessity for gastrectomy with lymph node dissection for patients with submucosal invasive gastric cancer. Br J Surg. 2001;88:444-9.

16. Gotoda T, Yanagisawa A, Sasako M, Ono H, Nakanishi Y, Shimoda $\mathrm{T}$, et al. Incidence of lymph node metastasis from early gastric cancer: estimation with a large number of cases at two large centers. Gastric Cancer. 2000;3:219-25.

17. Hatta W, Gotoda T, Oyama T, Kawata N, Takahashi A, Yoshifuku $\mathrm{Y}$, et al. Is radical surgery necessary in all patients who do not meet the curative criteria for endoscopic submucosal dissection in early gastric cancer? A multi-center retrospective study in Japan. J Gastroenterol. 2017;52:175-84.

18. Hatta W, Gotoda T, Oyama T, Kawata N, Takahashi A, Yoshifuku $\mathrm{Y}$, et al. A scoring system to stratify curability after endoscopic submucosal dissection for early gastric cancer. Am J Gastroenterol. 2017;112:874-81.

19. Sekiguchi M, Oda I, Taniguchi H, Suzuki H, Morita S, Fukagawa $\mathrm{T}$, et al. Risk stratification and predictive risk-scoring model for lymph node metastasis in early gastric cancer. J Gastroenterol. 2016;51:961-70.

20. Watanabe M, Miyata H, Gotoh M, Baba H, Kimura W, Tomita $\mathrm{N}$, et al. Total gastrectomy risk model: data from 20,011 Japanese patients in a nationwide internet-based database. Ann Surg. 2014;260:1034-9.

21. Kurita N, Miyata H, Gotoh M, Shimada M, Imura S, Kimura $\mathrm{W}$, et al. Risk model for distal gastrectomy when treating gastric cancer on the basis of data from 33,917 Japanese patients collected using a nationwide web-based data entry system. Ann Surg. 2015;262:295-303.

22. Yang JY, Lee HJ, Kim TH, Huh YJ, Son YG, Park JH, et al. Shortand long-term outcomes after gastrectomy in elderly gastric cancer patients. Ann Surg Oncol. 2017;24:469-77.

Publisher's Note Springer Nature remains neutral with regard to jurisdictional claims in published maps and institutional affiliations. 